\title{
Analysis of the false-negative results in endobronchial ultrasound-guided transbronchial needle aspiration
}

\author{
Yangli Liu ${ }^{1 *}$, Xiaoran Liu ${ }^{2 *}$, Fengjia Chen ${ }^{1}$, Huai Liao ${ }^{1 *}$ and Canmao Xie ${ }^{1 *}$ \\ ${ }^{1}$ The division of pulmonary and critical care medicine, The First Affiliated Hospital of Sun Yat-sen University, People's Republic of China \\ ${ }^{2}$ Emergency Department, The Affiliated Hospital of Hainan Medical University, People's Republic of China \\ \#These authors contributed equally to this work
}

\begin{abstract}
Background: To reduce the false-negative diagnosis of Endobronchial ultrasound-guided transbronchial needle aspiration (EBUS-TBNA) is a good way to improve its diagnostic efficiency. Few published studies focused on it. Therefore, we present our experience with EBUS-TBNA in an evaluation of the cases with a negative diagnosis in the procedure and analyze the factors that may limit the diagnostic yield.

Methods: EBUS-TBNA procedures performed at our institution between September 1, 2012 and May 30, 2016 for enlarged mediastinal or hilar lymphadenopathy were retrospectively analyzed. Negative EBUS-TBNA results were analyzed. Patient age, gender, disease species, sampling site distribution, lesion size, punctures number and operator experience were collected. Statistical analyses were performed with GraphPad InStat 3.05 software.

Results: A total of 328 patients underwent EBUS-TBNA for 533 lymph nodes during the study period, 64 patients got a negative result and the failure rate was 19.5\%. A significantly higher negative rate was shown in diagnosing lymphoma and benign disorders including tuberculosis, granuloma and nonspecific inflammation. Lesions of N1 (group 10,11) stations and with smaller size had a significantly lower sensitivity than those of mediastinal stations (group 2,3,4,7) and with larger size. After the first 20 procedures for learning, the physician could decrease the failure rate to about $20 \%$ in diagnosing both malignancy and benign diseases.
\end{abstract}

Conclusions: EBUS-TBNA has a higher false-negative rate in diagnosing lymphoma or benign disorders compared to malignancies, especially for lesions of N1 (group 10,11) stations and with smaller size by less skilled operators.

\section{Introduction}

Endobronchial ultrasound-guided transbronchial needle aspiration (EBUS-TBNA) is a real-time, accurate, minimally invasive, and safe technique for assessing undiagnosed mediastinal and hilar adenopathy [1], including diagnosing and staging of lung cancer [2], diagnosis of mediastinal malignancies as well as some specific benign diseases, such as sarcoidosis, tuberculosis (TB) and nonspecific inflammation.

The American College of Chest Physicians' (ACCP) lung cancer guidelines (third edition) reported an overall median sensitivity of $89 \%$ for mediastinal staging of lung cancer [3]. However, the diagnostic yield for benign disorders varied a lot in different studies [4,5]. In a recent meta-analysis of its use in sarcoidosis, the pooled sensitivity of EBUS-TBNA was 79\% [6]. As EBUS-TBNA became more available and adopted by clinicians, questions emerged about the optimal performance of the procedure and best conditions for a maximal diagnostic yield [7]. Besides the disease species, many technical aspects may infect the diagnostic yield of EBUS-TBNA including ultrasonographic features, with or without suction, needle size, feasibility of rapid on-site evaluation (ROSE), operators' skills.

To maximize the diagnostic rate, we should try to reduce the missed diagnosis and false negative diagnosis. Analyzing and summarizing the failure cases is a good way. However, few published studies focused on the cases with negative EBUS-TBNA biopsy results or analyzed the failure reason. Therefore, in this study, we present our experience with EBUS-TBNA in an evaluation of the cases who failed to get a positive diagnosis in the procedure and analyze the factors that may limit the diagnostic yield including disease species, lesion location and size as well as operators' experience, etc.

\section{Materials and methods}

We retrospectively reviewed all of the patients presented at our institution and underwent EBUS-TBNA from September 1, 2012 to May 30, 2016. Our study was approved by the Institutional Review Board of Sun Yat-Sen University. Patient age, gender, disease category, sampling site distribution, size and puncture number was collected. Abnormal lymph nodes were identified before the procedure based on chest CT scan appearance of enlarged mediastinal or hilar lymph nodes. In some cases, FDG-PET scans were evaluated to help identify nodes of interest.

EBUS-TBNA were performed under local anesthesia with a $2 \%$ lidocaine solution by a team of interventional pulmonologists.

${ }^{\star}$ Correspondence to: Canmao Xie, The division of pulmonary and critical care medicine, The First Affiliated Hospital of Sun Yat-sen University, Zhongshan Road, Guangzhou, Guangdong Province, PR China, E-mail: 21300282@qq.com

Huai Liao, The division of pulmonary and critical care medicine, The First Affiliated Hospital of Sun Yat-sen University, Zhongshan Road, Guangzhou, Guangdong Province, PR China, E-mail: liaohuai1208@hotmail.com

Key words: EBUS, TBNA, lymphadenopathy, negative results, failure rate

Received: August 06, 2018; Accepted: August 30, 2018; Published: September 06,2018 
Intravenous sedation was not performed before or during the bronchoscopy. A linear array ultrasonic bronchoscope (BF-UC-260FOL8, Olympus, Tokyo, Japan) with a dedicated 22-gauge needle (NA201SX-4022, Olympus) was used by the same team of bronchoscopists to perform the ultrasonic examination, transbronchial aspiration, and core biopsy. Each bronchoscopist determined the number of passes based on the amount of obtained biopsy material. Each TBNA specimen was aspirated from the needle onto a slide using air in the syringe. A smear was made with the help of another slide and it was immediately fixed in $95 \%$ alcohol. Other samples were fixed in a formalin solution for cell block processing and histological evaluation. Culture or special stains were based on clinical suspicion of infection. A rapid on-site cytology exam (ROSE) was not performed because of the lack of a cytologist or pathologist in our clinic center.

All aspirated specimens were categorized according to their pathological report. The presence of frank malignant cells indicated a malignant specimen, while obvious evidence of benign etiologies indicated a benign specimen. Samples that only showed blood, mucus, benign bronchial epithelial cells, or that contained no lymphoid tissue were considered inadequate samples. Other results of EBUS-TBNA were considered negative.

Negative EBUS-TBNA biopsy results were determined to represent false negatives in this study based on further confirmation made by mediastinoscopy, thoracoscopy, biopsy of a different lymph node station, repeated EBUS-TBNA, surgery or clinical and radiological follow-up (at least 2 years of follow-up) based on the clinical situation. Patients with loss of follow-up or with insufficient data to determine the final diagnosis were excluded. And the repeat EBUS-TBNA were not included in our analysis.

\section{Statistical analysis}

Data were analyzed with GraphPad InStat 3.05 software (GraphPad Software, San Diego, CA, USA) and are expressed as either group percentages (for the categorical variables) or as the mean \pm standard deviation (for the continuous variables). The $t$-test was applied to compare the continuous variables that exhibited a normal distribution, and the Mann-Whitney $U$ test was applied for the continuous variables that did not exhibit a normal distribution. A P-value $<0.05$ was considered statistically significant.

\section{Results}

\section{Demographic data}

We reviewed over 328 EBUS-TBNAs and identified 64 patients with negative results, the failure rate was $19.5 \%$. The mean age of the patients in the negative group was $56 \mathrm{y}$ and there were 42 male patients (65.6\%), EBUS-TBNA was carried out in 99 lymph nodes and 3masses. No serious complications associated with the EBUS-TBNA procedure were reported, and minor bleeding at the biopsy site was not considered a complication. The demographic and lesion characteristics of the total patients included in this study are summarized in table 1.

\section{Distribution of the undiagnosed diseases}

Analysis of the negative cases which were further confirmed by other ways of biopsy or clinical follow-up revealed a lower undiagnosed rate in malignancies including both lung cancer $12.1 \%(28 / 232)$ and extrathoracic malignancies $11.1 \%(1 / 9)$. However a high negative rate was shown in diagnosing Tuberculosis 33.3\% (14/42), Granuloma
$46.2 \%(6 / 13)$ and nonspecific inflammation $25.0 \%$ (5/20). EBUSTBNA failed to diagnose any of the cases of lymphoma, Interstitial lung diseases or Castleman diease. Overall, the failure rate was significantly higher in benign diseases than in malignancies ( $35.0 \%, 28 / 80$ vs. $14.5 \%$, 36/248) (Tables 2 and 3).

\section{Distribution of the negative biopsy site}

More than half of the EBUS-TBNA samples were obtained from the paratracheal, retrotracheal and subcarinal (group 2,3,4,7) lymph nodes and presented with a relatively low negative rate (group 2R:16.7\%, group $3: 11.8 \%$, group $4 \mathrm{R}: 13.0 \%$, group $4 \mathrm{~L}: 19.2 \%$, group $7: 18.5 \%$, respectively). In hilar and interlobar lymph nodes, EBUS-TBNA showed a relatively high undiagnosed rate: group 10R:22.8\%, group 10L:26.7\%, group 11R:28.9\% and group 11L:23.8\%, respectively. By dividing the lymph nodes into two groups, namely mediastinal (group 2,3,4,7) and N1 (group 10,11) stations, the negative rate of EBUSTBNA was $16.1 \%(53 / 330)$ vs.26.1\% (46/176), and this difference was highly significant $(\mathrm{P}<0.05)$ (Tables 3 and 4$)$.

\section{Lymph node size and number of needle passes}

To further explore the influence of the size and puncture number of per lymph node to the diagnosis efficiency of EBUS-TBNA, we compared the negative result group and the positive result group and found that the negative result group was with smaller lymph node size $(17.96 \pm 0.84 \mathrm{~mm}$ vs. $20.99 \pm 0.49 \mathrm{~mm}, \mathrm{P}<0.05)$. However, the number of needle passes per sampling site showed no significant difference between the two groups $(\mathrm{P}>0.05)$ (Table 5).

\section{Learning curve}

EBUS-TBNA yield varied with operator experience. There were 3 physicians in our clinic center who were able to complete the essential

Table 1. The demographic and lesion characteristics of the patients undergoing Endobronchial Ultrasound-Guided Transbronchial Needle Aspiration (EBUS-TBNA)

\begin{tabular}{|c|c|c|}
\hline Characteristics & Negative group & Total patients \\
\hline No. of patients & 64 & 328 \\
\hline Age, $y$ & $56 \pm 13$ & $55 \pm 13$ \\
\hline Male/Female & $42 / 22$ & $235 / 93$ \\
\hline Nodes + Masses & 99 nodes +3 masses & 506 nodes +27 masses \\
\hline
\end{tabular}

Table 2. Distribution of the undiagnosed diseases of EBUS-TBNA

\begin{tabular}{|c|c|c|c|}
\hline Disease species & Negative cases & Total cases & Failure rate \\
\hline Lung cancer & 28 & 232 & $12.1 \%$ \\
\hline Tuberculosis & 14 & 42 & $33.3 \%$ \\
\hline Granuloma & 6 & 13 & $46.2 \%$ \\
\hline Lymphoma & 6 & 6 & $100 \%$ \\
\hline Nonspecific inflammation & 5 & 20 & $25 \%$ \\
\hline Interstitial lung diseases & 2 & 2 & $100 \%$ \\
\hline Castleman diease & 1 & 1 & $100 \%$ \\
\hline Extrathoracic malignancy & 1 & 9 & $11.1 \%$ \\
\hline Benign tumor & 1 & 3 & $33.3 \%$ \\
\hline Total & 64 & 328 & $19.5 \%$ \\
\hline
\end{tabular}

Table 3. Failure rate by EBUS-TBNA in 328 Patients

\begin{tabular}{|c|c|c|}
\hline Characteristics & Failure rate & P value \\
\hline Disease species & & 0.0001 \\
\hline Malignancies & $14.5 \%(36 / 248)$ & \\
\hline Benign disorders & $35.0 \%(28 / 80)$ & 0.009 \\
\hline Lesion site & & \\
\hline Mediastinal (group 2,3,4,7) & $16.1 \%(53 / 330)$ & \\
\hline N1 (group 10,11) & $26.1 \%(46 / 176)$ & \\
\hline
\end{tabular}


Table 4. Distribution of the negative biopsy site

\begin{tabular}{|c|c|c|c|}
\hline Biopsy site & Negative number & Total number & Negative rate \\
\hline $\mathbf{2 R}$ & 3 & 18 & $16.7 \%$ \\
\hline $\mathbf{3}$ & 2 & 17 & $11.8 \%$ \\
\hline $\mathbf{4 R}$ & 16 & 123 & $13.0 \%$ \\
\hline $\mathbf{4 L}$ & 5 & 26 & $19.2 \%$ \\
\hline $\mathbf{7}$ & 27 & 146 & $18.5 \%$ \\
\hline $\mathbf{1 0 R}$ & 13 & 57 & $22.8 \%$ \\
\hline $\mathbf{1 0 L}$ & 4 & 15 & $26.7 \%$ \\
\hline $\mathbf{1 1 R}$ & 24 & 83 & $28.9 \%$ \\
\hline $\mathbf{1 1}$ & 5 & 21 & $23.8 \%$ \\
\hline Mass & 3 & 27 & $11.1 \%$ \\
\hline Total & 102 & 533 & $19.1 \%$ \\
\hline
\end{tabular}

Table 5. Difference of lymph node size and number of needle passes per sampling site between the negative result group and the positive result group

\begin{tabular}{|c|c|c|c|}
\hline Characteristics & $\begin{array}{c}\text { Negative group } \\
\text { (N=64) }\end{array}$ & $\begin{array}{c}\text { Positive group } \\
(\mathbf{N}=\mathbf{2 6 4})\end{array}$ & P \\
\hline Node size (mm) & $17.96 \pm 0.84$ & $20.99 \pm 0.49$ & 0.0072 \\
\hline No. of passes & $2 \pm 1$ & $2 \pm 1$ & 0.389 \\
\hline
\end{tabular}

steps of EBUS-TNBA and perform the procedure successfully with adequate tissue sampling. Figure 1 shows the curve of the failure rate of the procedures. After the first 20 procedures for learning, the physician could decrease the failure rate to about $20 \%$ in average in diagnosing both malignancy and benign diseases.

\section{Discussion}

EBUS-TBNA has become an important clinical tool for diagnosing unexplained mediastinal and hilar lymphadenopathy due to malignant or benign disease. Krasnik et al. first reported the utility of convex probe EBUS-TBNA in sampling mediastinal nodes in 2003 [8]. Subsequently, the diagnostic yield of EBUS-TBNA has been enhanced by performing rapid on-site cytological evaluations, increasing the number of lymph nodes sampled, and the use of a larger bore $21 \mathrm{G}$ needle [6]. In this retrospective study, EBUS-TBNA showed a satisfactory diagnostic yield with an overall sensitivity rate $80.5 \%$. We further analyzed the cases who failed to get a positive result by EBUS-TBNA in our clinic center and found that disease categories, details of sampling methodology including the location and size of lymph nodes, and experience of the bronchoscopists were important factors affecting the diagnostic yield of EBUS-TBNA.

Our study demonstrated an excellent diagnostic performance for EBUS-TBNA in detecting nodule malignancy, including both lung cancer and extrathoracic malignancies. The negative rate were only $12.1 \%(28 / 232)$ and $11.1 \%(1 / 9)$, respectively, similar to the results reported by Herth et al. [9] for EBUS-TBNA in detecting malignancy. However, EBUS-TBNA failed to diagnose any of the 6 cases of lymphoma who later required a surgical biopsy to confirm the diagnosis of lymphoma, which might be due to the use of cytological needles in EBUS-TBNA that compromised the maintenance of tissue architecture in the samples collected. Several authors [10,11] have criticized the use of EBUS-TBNA for some lymphoma subtypes, such as marginal zone lymphomas or hypo-cellular variants, due to the small volume of the samples that are collected. Few studies have investigated the capacity of EBUS-TBNA to diagnose benign disease. According to the results of the present study, EBUS-TBNA provided a significantly lower diagnostic yield for benign disorders including tuberculosis, sarcoidosis and nonspecific inflammation (failure rate was $33.3 \%$, $46.2 \%$ and $25.0 \%$ respectively). This could also be explained by the small tissue core specimen of EBUS-TBNA which might not reflect the whole LN view, only showing LN tissue/lymphocyte without obvious abnormal lesions or target LN not being involved in inflammation [12]. It is possible that the use of a $21 \mathrm{G}$ cytology needle with rapid on-site cytopathological support to collect an adequate sample could diminish the failure rate of EBUS-TBNA in investigations of the above diseases [13].

Both left (L) and right (R) lymph node stations are accessible for EBUS-TBNA, and these include: $2 \mathrm{R}, 2 \mathrm{~L}, 3,4 \mathrm{R}, 4 \mathrm{~L}, 7,10 \mathrm{R}, 10 \mathrm{~L}, 11 \mathrm{R}$, $11 \mathrm{~L}$, and 12R [14]. In our study, lymph node group 2,3,4,7,10,11 were all included and we found that hilar and interlobar lymph nodes (group 10,11) showed a significantly decreased diagnostic yield than paratracheal, retrotracheal and subcarinal (group 2,3,4,7) lymph nodes ( $73.9 \%$ vs. $83.9 \%$ ), which was in concert with previous study. Kazuhiro et al. [15] reported that the sensitivity of EBUS-TBNA to accurately diagnose the lymph nodes in group $10,11,12$ was $76.2 \%$, similar to that in our study. The rigid part on the tip and the outer diameter of the convex probe EBUS preventing visualization of lobar lymph nodes as well as the increased aspirate angle was thought to be the main reason that limited the diagnostic value of interlobar lymph nodes [15].

To further explore the influence of the size and aspiration number of lymph nodes to the diagnosis efficiency of EBUS-TBNA, we compared the negative result group and the positive result group and found that the negative result group was with smaller lymph node size $(17.96 \pm 0.84 \mathrm{~mm}$ vs. $20.99 \pm 0.49 \mathrm{~mm}, \mathrm{P}<0.05)$. It suggested that the size of lymph nodes was one of the key factors in determining the diagnostic accuracy of EBUS-TBNA. Similar conclusions have been detected in previous studies. Sun J et al. [16] reported that short-axis diameter of the lymph node was independent risk factor associated with positive pathology in diagnosing pulmonary sarcoidosis. Memoli JS et al. [17] found that larger lymph nodes are more likely to harbor metastases. Thus, it is reasonable for experienced bronchoscopists to choose the larger lymph node to obtain the highest yield for both benign and malignant diseases. However, the number of needle passes per sampling site showed no significant difference between the two groups ( $2 \pm 1$ vs. $2 \pm 1, \mathrm{P}>0.05)$, which was similar to the report of Ye et al. [18] who found that there was no significant association between the diagnostic accuracy of EBUS-TBNA and number of needle passes of lymph nodes in 101 patients with 225 lymph nodes sampled. Lee et al. [19] reported that sample adequacy was $90.1 \%$ after the first pass, $98.1 \%$ after two passes, and reached $100 \%$ after three passes in diagnosing lung cancer. However, no data exist regarding the number of needle passes required to obtain a sufficient diagnostic yield for lymphoma or other nonmalignant diseases of the mediastinum [7]. More studies focused on the relation of number of needle passes and the diagnostic yield in both benign and malignant diseases are needed.

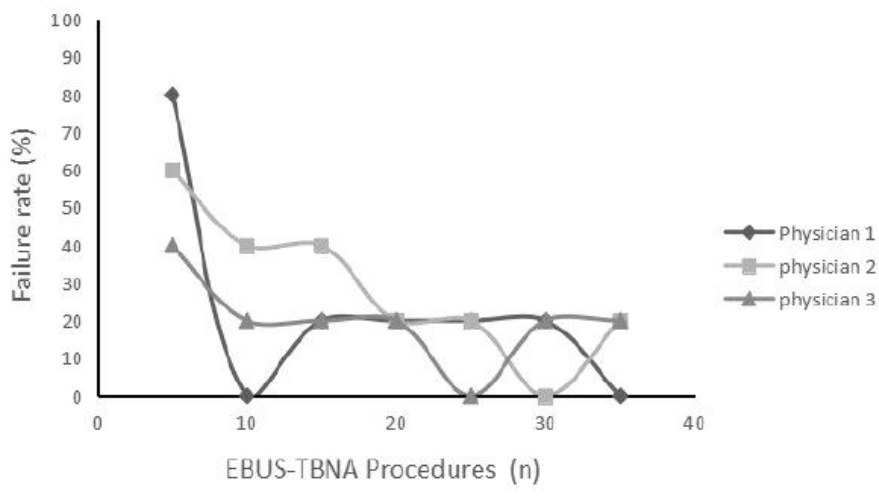

Figure 1. Learning curve for EBUS-TBNA. Failure rate steadily decreased with each procedure performed by attending physicians. After about 20 procedures, failure rate could decrease to an average of $20 \%$ 
The quality of EBUS-TBNA also depends on the skills and experience of the operator. Our study indicated that the diagnostic yield of EBUS improved with practice: after the first 20 procedures for learning, the physician could decrease the failure rate to an average of $20 \%$. Similarly, Wahidi et al. [20] found an average of 13 procedures was required to perform the first successful independent EBUS-TBNA for general pulmonary fellows. However, since there were only 3 bronchoscopists who had managed over 20 cases of EBUS-TBNA in our clinic center, the interpretation of the result was limited. Moreover, measurement of competency and procedural skill should include other skills, including simulation skill assessments and other performancebased metrics [7].

There were limitations associated with the current study. First, rapid on-site cytological evaluations were not available due to cost and limited manpower. The availability of rapid on-site cytopathologists would provide optimal specimen preparation and assessment to guide operator to get more specimens for further pathological examinations and microbiological tests $[12,21]$. Second, it was a retrospective study and included a relatively small number of patients with benign diseases like granuloma and tuberculosis, which might affect the diagnostic value analysis. Thirdly, the small specimen of EBUS-TBNA did not reflect the full pathology of the lesion and some patients received only clinical follow-up, not surgical diagnosis, which might lead to some misdiagnose. Thus, additional prospective clinical research with larger patient population is needed to improve the diagnostic value of EBUS-TBNA.

\section{Conclusions}

Endobronchial ultrasound-guided transbronchial needle aspiration is an accurate and safe procedure for diagnosing malignant mediastinal lymphadenopathy. However, it has a higher false-negative rate in diagnosing lymphoma or benign disorders compared to malignancies, especially for lesions of N1 (group 10,11) stations and with smaller size by less skilled operators. To decrease the false-negative rate, the indication for disease categories, location and size of the biopsy lesion, and experience of the operators should be better chosen.

\section{Funding}

This work was partly supported by grants from the National Science Foundation of China (NSFC) (No. 81260010, 81460006).

\section{Availability of data and material}

All data generated or analyzed during this study are included in this published article.

\section{References}

1. Varela-Lema L, Fernandez-Villar A, Ruano-Ravina A (2009) Effectiveness and safety of endobronchial ultrasound-transbronchial needle aspiration: a systematic review. Eur Respir J 5: 1156-1164. [Crossref]

2. Szlubowski A, Zielinski M, Soja J, Filarecka A, Orzechowski S, Pankowski J, et al. (2014) Accurate and safe mediastinal restaging by combined endobronchial and endoscopic ultrasound-guided needle aspiration performed by single ultrasound bronchoscope. Eur J Cardiothorac Surg 2: 262-266. [Crossref]
3. Silvestri GA, Gonzalez AV, Jantz MA, Margolis ML, Gould MK, et al. (2013) Methods for staging non-small cell lung cancer: Diagnosis and management of lung cancer, 3rd ed: American College of Chest Physicians evidence-based clinical practice guidelines. Chest 5: e211S-e250S. [Crossref]

4. Boujaoude Z, Dahdel M, Pratter M, Kass J (2012) Endobronchial ultrasound with transbronchial needle aspiration in the diagnosis of bilateral hilar and mediastinal lymphadenopathy. J Bronchology Interv Pulmonol 1: 19-23. [Crossref]

5. Madan K, Mohan A, Ayub II, Jain D, Hadda V, et al. (2014) Initial experience with endobronchial ultrasound-guided transbronchial needle aspiration (EBUS-TBNA) from a tuberculosis endemic population. J Bronchology Interv Pulmonol 3: 208-214. [Crossref]

6. Agarwal R, Srinivasan A, Aggarwal AN, Gupta D (2012) Efficacy and safety of convex probe EBUS-TBNA in sarcoidosis: a systematic review and meta-analysis. Respir Med 106: 883-892. [Crossref]

7. Wahidi MM, Herth F, Yasufuku K, Shepherd RW, Yarmus L, et al. (2016) Technical Aspects of Endobronchial Ultrasound-Guided Transbronchial Needle Aspiration: CHEST Guideline and Expert Panel Report. Chest 3: 816-835. [Crossref]

8. Krasnik M, Vilmann P, Larsen SS, Jacobsen GK (2003) Preliminary experience with a new method of endoscopic transbronchial real time ultrasound guided biopsy for diagnosis of mediastinal and hilar lesions. Thorax 12: 1083-1086. [Crossref]

9. Herth FJ, Eberhardt R, Krasnik M, Ernst A (2008) Endobronchial ultrasound-guided transbronchial needle aspiration of lymph nodes in the radiologically and positron emission tomography-normal mediastinum in patients with lung cancer. Chest 4: 887891. [Crossref]

10. Farmer PL, Bailey DJ, Burns BF, Day A, LeBrun DP (2007) The reliability of lymphoma diagnosis in small tissue samples is heavily influenced by lymphoma subtype. Am J Clin Pathol 3: 474-480. [Crossref]

11. Kheir F, Itani A, Assasa O, Alraiyes AH (2016) The utility of endobronchial ultrasoundtransbronchial needle aspiration in lymphoma. Endosc Ultrasound 1: 43-48. [Crossref]

12. Yang H, Wang S, Teng J, Han B (2018) Utility of endobronchial ultrasound-guided transbronchial needle aspiration in diagnosing non-specific inflammatory intrathorcacic lymphadenitis. Clin Respir J 2: 691-698. [Crossref]

13. Kennedy MP, Jimenez CA, Bruzzi JF, Mhatre AD, Lei X, et al. (2008) Endobronchia ultrasound-guided transbronchial needle aspiration in the diagnosis of lymphoma. Thorax 4: 360-365. [Crossref]

14. Szlubowski A, Kuzdzał J, Kołodziej M, Soja J, Pankowski J, et al. (2009) Endobronchial ultrasound-guided needle aspiration in the non-small cell lung cancer staging. Eur $J$ Cardiothorac Surg 35: 332-335. [Crossref]

15. Mahajan SS, Leko V, Simon JA, Bedalov A (2011) Sirtuin modulators. Handb Exp Pharmacol 206: 241-255. [Crossref]

16. Sun J, Yang H, Teng J, Zhang J, Zhao H, et al. (2015) Determining factors in diagnosing pulmonary sarcoidosis by endobronchial ultrasound-guided transbronchial needle aspiration. Ann Thorac Surg 2: 441-445. [Crossref]

17. Wang Memoli JS, El-Bayoumi E, Pastis NJ, Tanner NT, Gomez M, et al. (2011) Using endobronchial ultrasound features to predict lymph node metastasis in patients with lung cancer. Chest 6: 1550-1556. [Crossref]

18. Ye T, Hu H, Luo X, Chen H (2011) The role of endobronchial ultrasound guided transbronchial needle aspiration (EBUS-TBNA) for qualitative diagnosis of mediastinal and hilar lymphadenopathy: a prospective analysis. BMC Cancer 11: 100. [Crossref]

19. Lee HS, Lee GK, Lee HS, Kim MS, Lee JM, et al. (2008) Real-time endobronchial ultrasound-guided transbronchial needle aspiration in mediastinal staging of non-small cell lung cancer: how many aspirations per target lymph node station. Chest 2: 368 374. [Crossref]

20. Wahidi MM, Hulett C, Pastis N, Shepherd RW, Shofer SL, et al. (2014) Learning experience of linear endobronchial ultrasound among pulmonary trainees. Chest 3 : 574-578. [Crossref]

21. Nakajima T, Yasufuku K (2011) How I do it--optimal methodology for multidirectional analysis of endobronchial ultrasound-guided transbronchial needle aspiration samples. J Thorac Oncol 1: 203-206. [Crossref]

Copyright: (C2018 Liu Y. This is an open-access article distributed under the terms of the Creative Commons Attribution License, which permits unrestricted use, distribution, and reproduction in any medium, provided the original author and source are credited. 